Check for updates

Cite this: J. Mater. Chem. C, 2021 , 9, 12624

Received 30th April 2021,

Accepted 12th August 2021

DOI: $10.1039 / \mathrm{d} 1 \mathrm{tc0} 2004 \mathrm{~h}$

rsc.li/materials-c

\title{
SERS multiplexing of methylxanthine drug isomers via host-guest size matching and machine learning $\dagger$
}

\author{
Weng-I Katherine Chio, (D) abc Jia Liu, ${ }^{\text {ab }}$ Tabitha Jones, (D) ab Jayakumar Perumal, ${ }^{\mathrm{c}}$ \\ U. S. Dinish, (D) ${ }^{c}$ Ivan P. Parkin, (D) ${ }^{b}$ Malini Olivo ${ }^{c}$ and Tung-Chun Lee (D) *ab
}

\begin{abstract}
Multiplexed detection and quantification of structurally similar drug molecules, methylxanthine MeX, incl. theobromine TBR, theophylline TPH and caffeine CAF, have been demonstrated via solution-based surface-enhanced Raman spectroscopy (SERS), achieving highly reproducible SERS signals with detection limits down to $\sim 50 \mathrm{nM}$ for TBR and $\mathrm{TPH}$, and $\sim 1 \mu \mathrm{M}$ for CAF. Our SERS substrates are formed by aqueous self-assembly of gold nanoparticles (Au NPs) and supramolecular host molecules, cucurbit[n]urils ( $\mathrm{CB} n, n=7,8)$. We demonstrate that the binding constants can be significantly increased using a host-guest size matching approach, which enables effective enrichment of analyte molecules in close proximity to the plasmonic hotspots. The dynamic range and the robustness of the sensing scheme can be extended using machine learning algorithms, which shows promise for potential applications in therapeutic drug monitoring, food processing, forensics and veterinary science.
\end{abstract}

\section{Introduction}

Theobromine (TBR, 3,7-dimethylxanthine), theophylline (TPH, 1,3dimethylxanthine) and caffeine (CAF, 1,3,7-trimethylxanthine), which are structurally similar family members of purine alkaloids, are naturally present in foods and beverages such as chocolate, tea and coffee. Interestingly, TBR and TPH are two of the three major metabolites of CAF which coexist in blood plasma and urine. ${ }^{1,2}$ Methylxanthines (MeX) act as central nervous system stimulants for sustaining alertness by blocking adenosine receptors and inhibiting phosphodiesterases, ${ }^{3,4}$ while showing antitumoral and antiinflammatory properties. ${ }^{5}$ For instance, TBR and TPH are active ingredients of bronchodilator drugs taken to widen the airways in the lungs for asthma and other respiratory tract problems, ${ }^{6}$ whereas CAF is widely used in the formulations of prescription and over-thecounter medications. Although MeX are generally safe for human consumption except in the cases of severe overdose, ${ }^{7}$ they are potentially toxic to small animals such as cats and dogs. ${ }^{8}$

\footnotetext{
${ }^{a}$ Institute for Materials Discovery, University College London (UCL), London WC1H OAJ, UK. E-mail: tungchunlee@ucl.ac.uk

${ }^{b}$ Department of Chemistry, University College London (UCL), London WC1H OAJ, UK

${ }^{c}$ Translational Biophotonics Laboratory, Institute of Bioengineering and Bioimaging (IBB), Agency for Science Technology and Research (A*STAR), Singapore 138667, Singapore

$\dagger$ Electronic supplementary information (ESI) available: Limit of detection, NMR, computational details, UV-Vis, Raman, SERS and machine learning. See DOI: $10.1039 / \mathrm{d} 1 \mathrm{tc02004h}$
}

Development of a high-performance MeX sensor with multiplexing ability is thus crucial for effective diagnosis of caffeine intoxication (a.k.a caffeinism) ${ }^{2}$ and other diseases, therapeutic drug monitoring, quality control of consumer products in the food and pharmaceutical industries, as well as for forensics and veterinary science. Conventional methods for MeX detection are based on high-performance liquid chromatography, near infrared spectroscopy, immunoassay, voltammetry and fluorescence, ${ }^{6,9-17}$ but not all of these methods allow multiplexed detection of MeX in real-time with minimal sample preparation and high performance.

Surface-enhanced Raman spectroscopy (SERS) is an analytical technique capable of quantitatively discriminating multiple structurally similar analyte molecules located in close proximity to the plasmonic nanostructures (e.g. Au NPs and Ag NPs) via their vibrational fingerprints, with additional advantages such as rapid response, high sensitivity, selectivity and reproducibility. Surprisingly, most of the previous SERS studies on MeX detection were based on $\mathrm{Ag} \mathrm{NPs}^{18-27}$ for their relatively strong signals despite Au NPs having higher chemical stability, reproducibility and biocompatibility. ${ }^{28}$ This is probably due to the poor sensitivity and reproducibility of the SERS signals resulted from bare Au NPs or uncontrolled aggregation triggered by $\mathrm{NaCl}^{1,29}$ The interparticle spacing between Au NPs can be precisely controlled via cucurbit $[n]$ uril $(\mathrm{CB} n)$ mediated aggregation, thus localising the analyte molecules at the centre of or in close proximity to the plasmonic hotspots via formation of host-guest complexes. ${ }^{30-36}$ Previous work on MeX detection 
mainly focuses on CAF, while TBR and TPH, in particular their multiplexed SERS sensing, remain largely unexplored.

Herein, the host-guest complexations between $\mathrm{CB} n(n=7$, 8) and structurally similar drug molecules, MeX (TBR, TPH and CAF), were investigated in solution as to quantify their key binding parameters. While precedent examples of $\mathrm{Au}$ NP: CB SERS systems mostly focus on using CB7 as the supramolecular host, the use of the larger homologue CB8 as the host which can effectively encapsulate larger biomolecules, i.e. MeX in our case, is relatively rare. In particular, we showed a significant increase in binding constants by matching the molecular size of the host-guest pair (Fig. 1 and 2). Quantitative SERS detection of MeX was demonstrated with highly reproducible signals via formation of precise plasmonic hotspots within the Au NP: CB nanoaggregates. Thanks to the strong surface enrichment effect of CBs, the detection limit of CAF is down to $\sim 1 \mu \mathrm{M}$ while those of its demethylated analogues, TBR and TPH, have reached $\sim 50 \mathrm{nM}$, which is at least an order of magnitude better than other similar SERS techniques in the literature (see Table S1 for details, ESI $\dagger)^{1,20-22,26,27}$ and covers the lower limit of MeX in human urine samples. ${ }^{2}$ Notably, multiplexed detection of TBR and TPH at sub- $\mu \mathrm{M}$ concentrations was also demonstrated with CB8 using our SERS sensing platform. Finally, we showed that the dynamic range and the robustness of our sensing scheme can be further enhanced by machine learning algorithms, partial least squared regression (PLSR) and artificial neural networks (ANNs), which effectively modelled the signal non-linearity at higher analyte concentrations.

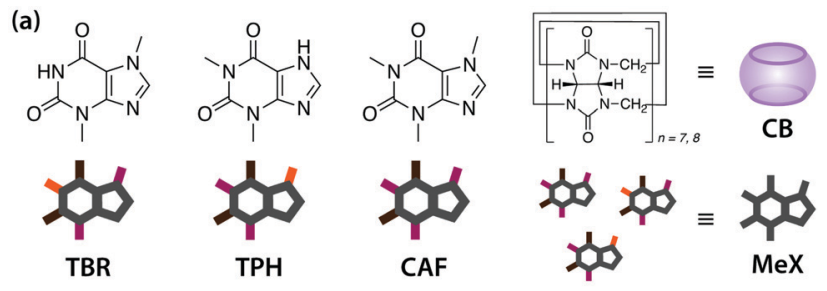

(b)
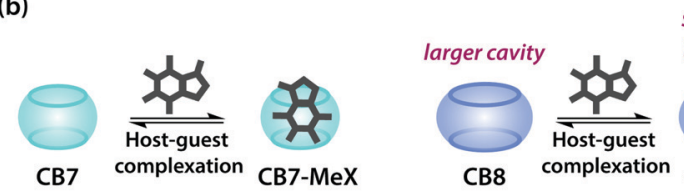

stronger binding

(c)

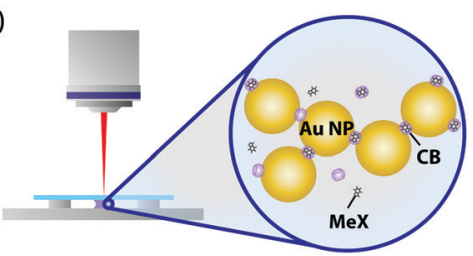

Fig. 1 Schematic illustrations of (a) MeX (TBR, TPH and CAF) and CB, (b) the formation of 1:1 CB7-MeX and CB8-MeX host-guest complexes, (c) the SERS measurement of the Au NP-CB-MeX sample solution. (a)

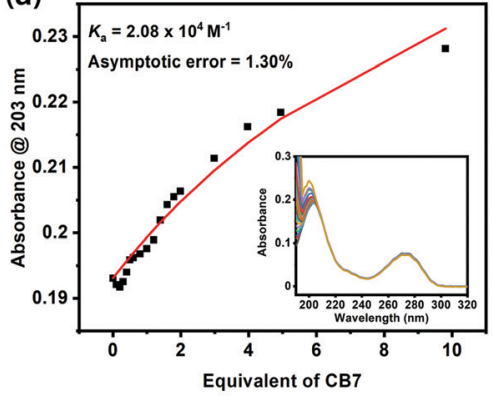

(c)

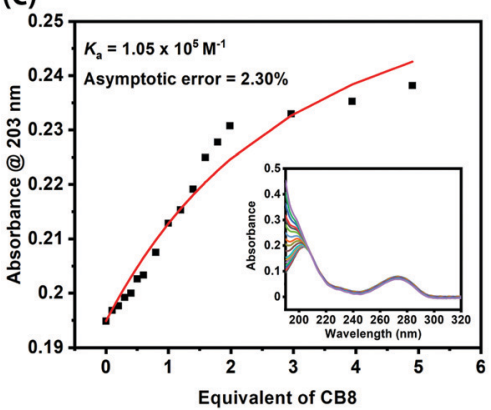

(b)

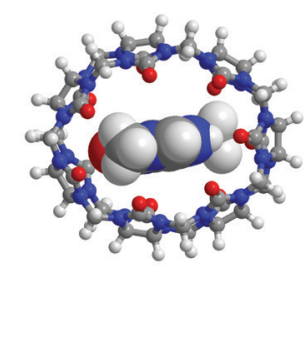

(d)

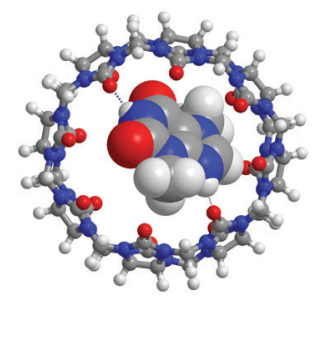

Fig. 2 (a) UV-Vis titration of $4 \mu M$ TBR with CB7. Inset: UV-Vis spectra of CB7-TBR complex. (b) DFT-optimised molecular model of the CB7-TBR complex. (c) UV-Vis titration of $4 \mu \mathrm{M}$ TBR with CB8. Inset: UV-Vis spectra of CB8-TBR complex. (d) DFT-optimised molecular model of the CB8TBR complex. The binding curves were fitted by assuming 1:1 binding model from which the binding constants were derived. DFT models were optimised at CPCM/wB97X-D/6-31G* level of theory.

\section{Materials and methods}

\section{Materials}

$40 \mathrm{~nm}$ citrate-stabilised gold nanoparticles (Au NPs) were purchased from nanoComposix. Paraformaldehyde, $\mathrm{HCl}$, theobromine (TBR), theophylline (TPH) and caffeine (CAF) were purchased from Sigma-Aldrich. Methanol and ethanol were purchased from VWR. Glycoluril was purchased from Acros Organics. Cucurbit $[n]$ urils ( $\mathrm{CB} n, n=7-8)$ were synthesised and isolated according to literature. ${ }^{37}$ All chemicals were used as received without further purification. 18.2 M $\Omega$ Milli-Q water was used in all experiments.

\section{Nuclear magnetic resonance (NMR) spectroscopy}

$1 \mathrm{mM}$ of CB7-TBR, CB7-TPH and CB7-CAF solutions were prepared in $\mathrm{D}_{2} \mathrm{O}$ with $1: 1$ molar ratio. Similarly, $1 \mathrm{mM}$ of CB8-TBR, CB8-TPH and CB8-CAF solutions were prepared in $10 \mathrm{mM}$ of DCl with 1:1 molar ratio. ${ }^{1} \mathrm{H}$ NMR spectra were measured using a Bruker Avance III 400 spectrometer. Chemical shifts (in ppm) were referenced to $\mathrm{D}_{2} \mathrm{O}$ with $\delta=$ 4.80 ppm for ${ }^{1} \mathrm{H}$ NMR.

\section{UV-Visible spectroscopy}

UV-Vis measurements were performed on Agilent Cary 500 UVVis-NIR spectrophotometer in a cuvette with $10 \mathrm{~mm}$ optical path length. For CB7-MeX binding studies, a small volume of 2 mM CB7 solution was added to $4 \mu \mathrm{M}$ MeX solution sequentially 
up to 5 or more equivalents. For CB8-MeX binding studies, a small volume of $1 \mathrm{mM}$ CB8 solution (pre-dissolved in $10 \mathrm{mM} \mathrm{HCl}$ ) was added to $4 \mu \mathrm{M}$ MeX solution (pre-dissolved in $10 \mathrm{mM} \mathrm{HCl}$ ) up to 2 or more equivalents. The concentrations of CB7 and CB8 were increased gradually while those of MeX were kept approximately constant. Prior to fitting, proportionated absorbance of CB7 and CB8 were subtracted accordingly to eliminate the effect that might be caused by their UV-Vis absorption around $190 \mathrm{~nm}$. Additionally, spectra for samples containing CB8 were aligned by setting the absorbance at $500 \mathrm{~nm}$ equal to zero to minimise the effect of turbidity at high equivalents of CB8.

\section{Simulations}

Density functional theory (DFT) calculations were performed using Gaussian and Spartan 18 Parallel Suite. Force-field calculations were performed using Chem3D. Geometry optimisation was first performed using MMFF94, followed by full optimisation at the required level of theory, in our case wB97XD/6-31G* followed by CPCM/wB97X-D/6-31G*. Restricted (closed-shell) models were used in all quantum mechanical calculations. The binding energies of the $[\mathrm{CB}-\mathrm{MeX}-\mathrm{H}]^{+}$inclusion complexes were calculated from the energy difference between the complex and the total energies of $\mathrm{CB}$ and $[\mathrm{MeX}-\mathrm{H}]^{+}$which were optimised and calculated at the same level of theory.

\section{Raman and surface-enhanced Raman spectroscopy (SERS)}

Raman and SERS spectra were acquired using a Reinshaw Raman InVia Microscope with a $633 \mathrm{~nm}$ He-Ne laser (9.3 $\mathrm{mW})$. The laser was focused onto the sample via a $50 \times$ objective lens (N.A. $=0.75$ ). The grating used was 1800 lines $\mathrm{mm}^{-1}$ which gave a spectral resolution of $1 \mathrm{~cm}^{-1}$. All spectra were calibrated with respect to $\mathrm{Si}$ and acquired at room temperature. Stock solutions were prepared by mixing CB and MeX. For CB7 studies, $1 \mathrm{~mL}$ of the TBR stock solution with varying concentration of $0-0.2 \mathrm{mM}$ was added to $1 \mathrm{~mL}$ of a $0.2 \mathrm{mM}$ CB7 solution in a $2 \mathrm{~mL}$ Eppendorf tube. $20 \mu \mathrm{L}$ of the stock solution was then added to a $180 \mu \mathrm{L}$ Au NP solution in a $0.5 \mathrm{~mL}$ tube to give a final TBR concentration of $0-10 \mu \mathrm{M}$. For CB8 studies, $1 \mathrm{~mL}$ of the TBR stock solution with varying concentration of 0-0.1 mM was added to $1 \mathrm{~mL}$ of a $0.1 \mathrm{mM}$ CB8 solution (prepared in $10 \mathrm{mM} \mathrm{HCl}$ ) in a $2 \mathrm{~mL}$ Eppendorf tube. $20 \mu \mathrm{L}$ of the stock solution was then added to a $180 \mu \mathrm{L}$ Au NP solution in a $0.5 \mathrm{~mL}$ tube to give a final TBR concentration of $0-5 \mu \mathrm{M}$. Similar procedures were used to prepare sample solutions of TPH and CAF. The sample solution was vortexed for $30 \mathrm{~s}$ before dropping $15 \mu \mathrm{L}$ onto a custommade sample holder for SERS measurements. Three accumulations of $30 \mathrm{~s}$ scan were acquired on each measurement and five measurements were taken across different regions of interest per sample. The spectra were averaged and baseline corrected using an asymmetric least squares plugin in Origin.

\section{Results and discussion}

\section{Supramolecular chemistry of CB and MeX}

The conjugated acids of theobromine (TBR) and theophylline $(\mathrm{TPH}),[\mathrm{TBR}-\mathrm{H}]^{+}$and $[\mathrm{TPH}-\mathrm{H}]^{+}$, have $\mathrm{pKa}$ of 10.0 and 8.8 respectively, ${ }^{4}$ implying that they exist in their protonated forms in solution under our experimental condition. Caffeine (CAF) also exists in its protonated form in our studies as its conjugated acid has a $\mathrm{p} K_{\mathrm{a}}$ of $10.4 .{ }^{4}$ The binding of CB7 to CAF was first suggested by Issacs and co-workers in 2009 but without reporting the binding constant, ${ }^{38}$ meanwhile the host-guest complexations of CB with TBR and TPH were studied for the first time.

When CB7 and TBR were mixed in $\mathrm{D}_{2} \mathrm{O}$ with $1: 1$ stoichiometry, characteristic upfield shifts of the TBR proton signals $\left(H_{\mathrm{b}}\right.$ and $H_{\mathrm{c}}$ ) were observed in the ${ }^{1} \mathrm{H}$ NMR spectra, verifying the formation of host-guest complexes between CB7 and TBR (Fig. S1b, ESI $\dagger$ ). In particular, the NMR signal of $H_{\mathrm{b}}$ was significantly shifted upfield and broadened after host-guest complexation $\left(\Delta \delta_{\mathrm{b}}=-0.099 \mathrm{ppm}\right)$, indicating that $H_{\mathrm{b}}$ is located deep inside the CB7 cavity and that the binding kinetics fall into the intermediate exchange regime on the NMR time scale at $298 \mathrm{~K}$, respectively. This observation also implies that the $H_{\mathrm{a}}$ signal may be too broad to be observed in the NMR spectra. In a separate measurement, TBR was mixed with the larger CB homologue, CB8, in $10 \mathrm{mM} \mathrm{DCl}$ with $1: 1$ stoichiometry. Characteristic upfield shifts of the TBR proton signals $\left(H_{\mathrm{a}}, H_{\mathrm{b}}\right.$ and $H_{\mathrm{c}}$ ) were observed in the NMR spectra, verifying the formation of 1:1 CB8-TBR host-guest complexes (Fig. S2b, $\mathrm{ESI} \dagger) . \Delta \delta$ for $H_{\mathrm{a}}\left(\Delta \delta_{\mathrm{a}}=-0.014 \mathrm{ppm}\right)$ is greater than that for $H_{\mathrm{c}} / H_{\mathrm{b}}\left(\Delta \delta_{\mathrm{c}}=-0.010 \mathrm{ppm}\right.$ and $\left.\Delta \delta_{\mathrm{b}}=-0.011 \mathrm{ppm}\right)$ which indicates $H_{\mathrm{a}}$ is deeper inside the CB8 cavity, whereas $H_{\mathrm{c}} / H_{\mathrm{b}}$ are closer to the carbonyl portals. Compared to the CB7 case, the smaller upfield shift can be attributed to the larger cavity of CB8 which exhibits a weaker shielding effect. Meanwhile all proton signals appear to be in the fast exchange regime, which is consistent to the wider portal and thus weaker constrictive binding of CB8.

Although TPH and TBR are isomers, strong broadening of proton signals after host-guest complexation between $\mathrm{CB}$ and TPH was not observed in the ${ }^{1} \mathrm{H}$ NMR spectra (Fig. S3b, ESI $\dagger$ ). When $\mathrm{CB} 7$ and $\mathrm{TPH}$ were mixed in $\mathrm{D}_{2} \mathrm{O}$ with $1: 1$ stoichiometry, characteristic shifts of the TPH proton signals $\left(H_{\mathrm{a}}, H_{\mathrm{b}}\right.$ and $\left.H_{\mathrm{c}}\right)$ were observed, verifying the host-guest complexation between CB7 and TPH. In particular, $H_{\mathrm{c}} / H_{\mathrm{b}}\left(\Delta \delta_{\mathrm{c}}=-0.003 \mathrm{ppm}\right.$ and $\Delta \delta_{\mathrm{b}}=$ $-0.002 \mathrm{ppm})$ were upfield shifted whereas $H_{\mathrm{a}}$ was downfield shifted $\left(\Delta \delta_{\mathrm{a}}=0.021 \mathrm{ppm}\right)$ after host-guest complexation, implying $H_{\mathrm{c}} / H_{\mathrm{b}}$ should be within the CB7 cavity while $H_{\mathrm{a}}$ should be around the portal region. Notably, the host-guest complexation of CB8-TPH is very similar to that of CB7-TPH (Fig. S4b, $\mathrm{ESI} \dagger)$.

On the other hand, the relatively bulky MeX, CAF, shows smaller upfield shifts of the proton signals when mixing with $\mathrm{CB} 7$ in $\mathrm{D}_{2} \mathrm{O}$ with $1: 1$ stoichiometry (Fig. S5b, ESI $\dagger$ ), suggesting weaker binding of CAF to CB7. Interestingly, splitting of the NMR signal of $H_{\mathrm{d}}$ was observed after host-guest complexation, resulting in two different values for the change in chemical shift $(\Delta \delta)$ for $H_{\mathrm{d}}\left(\Delta \delta_{\mathrm{d}}=-0.002 \mathrm{ppm}\right.$ and $\left.-0.010 \mathrm{ppm}\right) . \Delta \delta$ for $H_{\mathrm{d}}$ is greater than that for $H_{\mathrm{c}} / H_{\mathrm{b}} / H_{\mathrm{a}}\left(\Delta \delta_{\mathrm{c}}=-0.002 \mathrm{ppm}, \Delta \delta_{\mathrm{b}}=\right.$ $-0.002 \mathrm{ppm}$ and $\Delta \delta_{\mathrm{a}}=-0.003 \mathrm{ppm}$ ) which indicates $H_{\mathrm{d}}$ is deep inside the $\mathrm{CB} 7$ cavity, whereas $H_{\mathrm{c}} / H_{\mathrm{b}} / H_{\mathrm{a}}$ are much closer to the 
carbonyl portal. Similar results were observed in the ${ }^{1} \mathrm{H}$ NMR spectra when CAF was mixed with CB8 with $1: 1$ stoichiometry (Fig. S6b, ESI $\dagger$ ).

Binding constants of the CB7-TBR and CB8-TBR complexes were obtained from UV-Vis titrations by fitting 1:1 binding models (Fig. 2a and c). Notably, the binding constant of CB8TBR $\left(1.05 \times 10^{5} \mathrm{M}^{-1}\right)$ was found to be five times larger than that of CB7-TBR $\left(2.08 \times 10^{4} \mathrm{M}^{-1}\right)$. Similar results were observed for TPH while the binding between CAF and CB8 is on par with that of CB7 (Fig. S3-S8, ESI $\dagger$ ). We note that it remains challenging to minimise the error in the fitting, despite multiple attempts in UV-Vis titration, due to the interfering UV absorption of CBs at $\sim 190 \mathrm{~nm}$ and, in the case of CB8, the effect of turbidity at high $\mathrm{CB}$ equivalents. Meanwhile the change in absorbance for the peak of MeX at $\sim 275 \mathrm{~nm}$ is too small to be precisely measured. Nevertheless, the fitted binding constants are considered sufficient for qualitative comparison in our context. Accurate binding constant data might be obtained using isothermal titration calorimetry (ITC). However, ITC measurements could not be performed due to unavailability of the instrument but could be a potential approach for further study. In particular, the generally larger binding constants of CB8-MeX complexes can be rationalised by host-guest size matching effects as described in the Rebek's 55\% rule, where a packing coefficient (PC) of 55\% gives the best binding affinity for host-guest complexes in solution, with a lower or higher PC resulting in a lower binding affinity. ${ }^{39}$ For instance, the packing coefficients of CB7-MeX are $0.70-0.78$ while those of CB8-MeX are $0.46-0.51$ (see Table $\mathrm{S} 2$ for details, ESI $\dagger$ ). Indeed upon binding with TBR, the molecular shape of CB7 becomes highly distorted and thus conformationally destabilised, whereas CB8 retains a round conformation resembling that of the ground state of its empty form (Fig. 2b and d).

Energy-minimised molecular models of the [CB7-MeX-H $]^{+}$ and $[\mathrm{CB} 8-\mathrm{MeX}-\mathrm{H}]^{+}$complexes calculated based on density functional theory (DFT) at CPCM/wB97X-D/6-31G* level of theory support the host-guest binding geometries derived from NMR (Fig. S1a-S6a, ESI $\dagger$ ). The dispersion-corrected DFT functional wB97X-D was chosen to accurately estimate the van der Waals interactions, which were expected to contribute significantly to the stability of these complexes. Implicit water model CPCM was selected to effectively account for the solvation energy of the protonated guests and complexes with a formal charge of +1 . Moreover, the binding energies of the $[\mathrm{CB}-\mathrm{MeX}-\mathrm{H}]^{+}$complexes (see Table S3 and S4 for details, ESI $\dagger$ ) are consistent to similar host-guest complexes reported in literature. ${ }^{34,40}$ TBR, TPH and CAF are fully or almost fully encapsulated within the cavity of CB7 and CB8, leaving both of the CB portals available for binding to the surface of Au NPs and thus localising them at the centre of or in close proximity to the plasmonic hotspots, which is critical to the subsequent SERS studies.

\section{Raman spectroscopy of MeX and SERS of CB}

The Raman spectrum of TBR powder is characterised by two major peaks at $622 \mathrm{~cm}^{-1}$ and $1334 \mathrm{~cm}^{-1}$, which are attributed to $\mathrm{C}=\mathrm{C}-\mathrm{C}$ deformation and imidazole ring stretching vibration respectively (Fig. S9a, ESI $\dagger$ ). ${ }^{41,42}$ The Raman spectrum of TPH powder is characterised by a main peak at $557 \mathrm{~cm}^{-1}$, which corresponds to pyrimidine ring deformation $+\mathrm{C}-\mathrm{N}-\mathrm{C}$ deformation $+\mathrm{CH}_{3}$ rocking (Fig. $\mathrm{S} 9 \mathrm{~b}, \mathrm{ESI} \dagger$ ). The other peaks at $668 \mathrm{~cm}^{-1}$ and $1316 \mathrm{~cm}^{-1}$ are assigned to $\mathrm{O}=\mathrm{C}-\mathrm{N}$ deformation + pyrimidine imidazole ring deformation and imidazole ring stretching vibration, respectively. ${ }^{41}$ Similarly, the Raman spectrum of CAF powder is characterised by two major peaks at $558 \mathrm{~cm}^{-1}$ and $1330 \mathrm{~cm}^{-1}$, corresponding to pyrimidine ring deformation $+\mathrm{C}-$ $\mathrm{N}-\mathrm{C}$ deformation $+\mathrm{CH}_{3}$ rocking and imidazole ring stretching vibration respectively (Fig. S9c, ESI $\dagger$ ). ${ }^{26,41}$

The two characteristic Raman peaks of CB7 at $444 \mathrm{~cm}^{-1}$ and $833 \mathrm{~cm}^{-1}$, which correspond to ring scissor and ring deformation modes, were clearly observed in the SERS spectra of CB7 at $447 \mathrm{~cm}^{-1}$ and $833 \mathrm{~cm}^{-1}$ (Fig. S9d, ESI $\dagger$ ). The slight shifts in peak position and peak broadening could be due to the solution effect and the molecular interaction between Au NPS and CB7. Notably, the ring scissor mode of CB8 is at a slightly lower wavenumber of $444 \mathrm{~cm}^{-1}$ whereas its ring deformation mode is at a slightly higher wavenumber of $834 \mathrm{~cm}^{-1}$ than that of $\mathrm{CB} 7$, which is consistent with the previous report. ${ }^{43}$

\section{SERS sensing of CB-MeX host-guest complexes}

The SERS detection of MeX was first performed with CB7 and TBR by adding a pre-mixed CB7-TBR solution into a $40 \mathrm{~nm}$ citrate-capped Au NP solution. The concentration of CB7 in the final mixture was kept constant at $10 \mu \mathrm{M}$ for all cases in order to ensure the formation of reproducible nanoaggregates, i.e. SERS substrates, since the aggregation kinetics is delicately determined by the ratio of Au NP: CB. ${ }^{34}$ As CB7 defines precise nanojunctions between Au NPs while TBR is fully or almost fully encapsulated within the CB7 cavity, TBR is localised at the plasmonic hotspots within the Au NP: CB7 nanoaggregates (Fig. S10a, ESI $\dagger$ ). It should be noted that no aggregation of $\mathrm{Au}$ NPs can be triggered, and no SERS signals can be observed in the absence of CB7 (Fig. S10b, ESI $\dagger$ ), thus illustrating the importance of CB on TBR sensing. In the Au NP: CB7 system, the characteristic Raman peak of TBR at $1312 \mathrm{~cm}^{-1}$, which is attributed to imidazole ring stretching vibration, can be clearly observed in the SERS spectra down to $0.5 \mu \mathrm{M}$ (Fig. S10c and d, ESI $\dagger)$. A strong linear correlation $\left(R^{2}>0.99\right)$ between the SERS intensity and concentration of TBR (up to $2 \mu \mathrm{M}$ ) was found, while the full range was fitted well by power law $\left(R^{2} \sim 0.94\right)$ (Fig. S10e, ESI $\dagger$ ).

Notably, the sensitivity of our SERS system for TBR detection can be significantly enhanced by using the larger CB homologue, i.e. CB8. The SERS detection of TBR was performed by adding a pre-mixed CB8-TBR solution into the Au NP solution, at a constant CB8 concentration of $5 \mu \mathrm{M}$, to form precise plasmonic nanojunctions as in the case of CB7 (Fig. 3a). It is noted that the pre-mixed CB8-TBR solution contained $10 \mathrm{mM}$ $\mathrm{HCl}$ to facilitate the dissolution of CB8. As expected, no aggregation or SERS signals can be observed in the absence of CB8 (Fig. 3b). The detection limit of TBR was found to be 10fold lower (i.e. down to $\sim 50 \mathrm{nM}$ ) in the Au NP: CB8 system (Fig. 3c and d), which is the lowest among all similar SERS 
(a) (b)

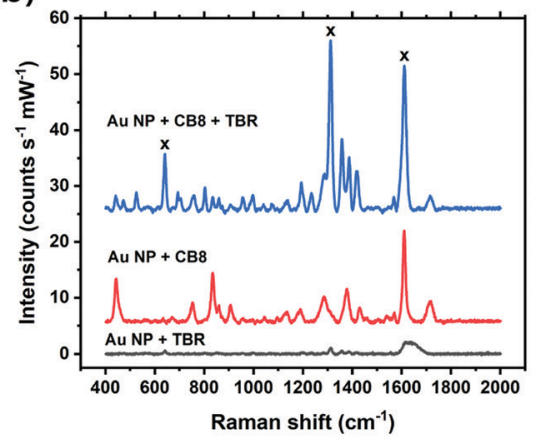

(c)

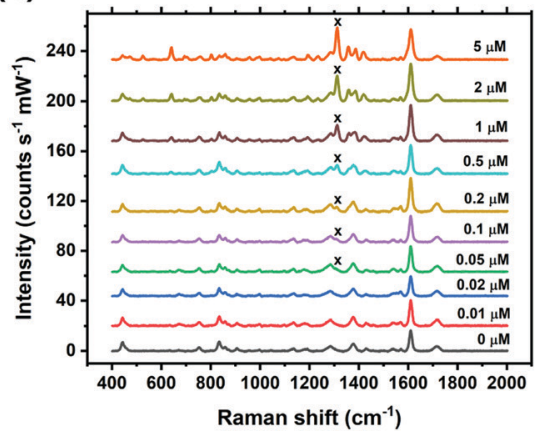

(d)

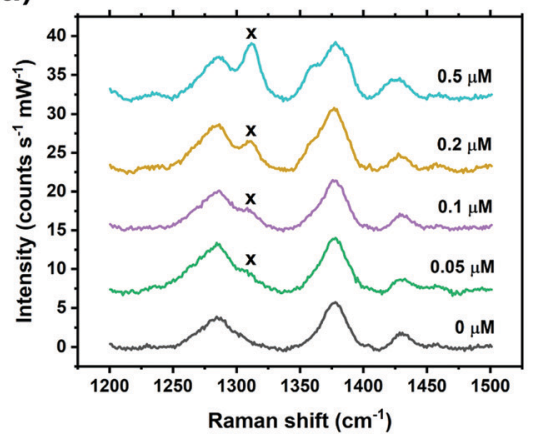

(e)

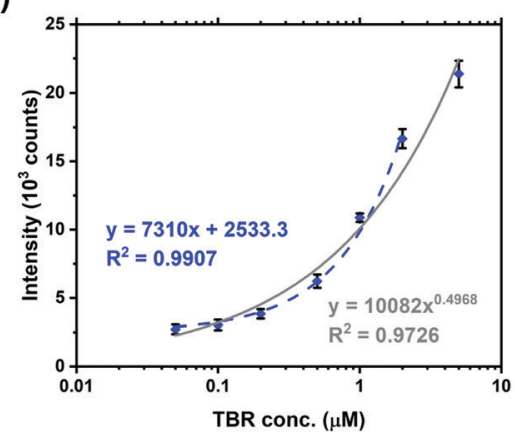

Fig. 3 (a) Schematic illustration of the precise plasmonic hotspots within Au NP: CB8 nanoaggregates for TBR detection (not to scale). (b) SERS spectra of $5 \mu \mathrm{M}$ TBR in the presence or absence of CB8. (c) Full-range and (d) zoom-in SERS spectra of TBR with different concentrations from 0 to $5 \mu M$. Main Raman peak of TBR at $1312 \mathrm{~cm}^{-1}$ is marked by $\mathrm{x}$. Spectra were baseline corrected and offset for clarity. (e) Corresponding plot of SERS intensity of the main TBR peak (marked by $x$ in (d)) against TBR concentration. Note: $x$-axis is plotted in log-scale to even out the spread of the data points for better illustration. The linear region at low concentration had been identified and fitted linearly, while the full range fitted well by power law.

platforms in the literature, ${ }^{1,20}$ with a strong linear correlation $\left(\begin{array}{ll}R^{2} & \sim 0.99\end{array}\right)$ between the SERS intensity and concentration of TBR up to $2 \mu \mathrm{M}$ (Fig. 3e). The full range was also fitted by power law with a good correlation $\left(R^{2} \sim 0.97\right)$.

The SERS detection of the isomer of TBR, i.e. TPH, was subsequently investigated in our studies, at a constant concentration of CB7 or CB8. Similarly, TPH is fully or almost fully encapsulated within the cavity of CB7 and CB8 as in the case of TBR while no aggregation of Au NPs can be triggered in the absence of CB (Fig. 4a, b and Fig. S11a, b, ESI†). The characteristic Raman peak of TPH at $573 \mathrm{~cm}^{-1}$, which corresponds to pyrimidine ring deformation $+\mathrm{C}-\mathrm{N}-\mathrm{C}$ deformation $+\mathrm{CH}_{3}$ rocking, can be clearly observed in the SERS spectra down to $50 \mathrm{nM}$ in the presence of CB7 (Fig. S11c and d, ESI $\dagger$ ), with a strong linear correlation $\left(R^{2} \sim 0.99\right)$ between the SERS intensity and concentration of TPH up to $2 \mu \mathrm{M}$ and a good correlation $\left(R^{2} \sim 0.98\right)$ for the full range fitted by power law (Fig. S11e, ESI $\dagger$ ). Interestingly, the detection limit of TPH in the Au NP: CB7 SERS system is 10-fold better than that of its isomer, TBR, despite being less Raman-active (Fig. S9a and b, ESI $\dagger$ ). This could be due to the difference in the binding geometries of the two complexes. Moreover, the $[\mathrm{CB} 8-\mathrm{TPH}-\mathrm{H}]^{+}$complex has a similar binding geometry to that of the $[\mathrm{CB} 7-\mathrm{TPH}-\mathrm{H}]^{+}$complex, with a detection limit of $\sim 0.1 \mu \mathrm{M}$ (Fig. $4 \mathrm{c}$ and d) and a very strong linear correlation $\left(R^{2} \sim 0.98\right)$ between the SERS intensity and concentration of TPH from 0.1 to $2 \mu \mathrm{M}$ (Fig. 4e). It should be noted that the $5 \mu \mathrm{M}$ data point deviates from the trendline, probably due to the LSPR peak shifting away from the $633 \mathrm{~nm}$ excitation or the difference in $\mathrm{pH}$.

The SERS detection of CAF was also investigated in our studies as control experiments (Fig. S12 and S13, ESI $\dagger$ ). Similar to the case of other MeX, no aggregation of Au NPs can be triggered in the absence of CB (Fig. S12b and S13b, ESI $\dagger$ ). The SERS signals of CAF are relatively weaker than those of its demethylated analogues, TBR and TPH, probably due to the nature of its bulkier size. The characteristic Raman peak of CAF at $1326 \mathrm{~cm}^{-1}$, which is attributed to imidazole ring stretching vibration, can be observed down to $5 \mu \mathrm{M}$ in the Au NP: CB7 system (Fig. S12c and d, ESI $\dagger$ ) and $1.25 \mu \mathrm{M}$ in the Au NP: CB8 nanoaggregates respectively (Fig. S13c and $\mathrm{d}$, ESI $\dagger$ ). A good correlation $\left(R^{2} \sim 0.98\right)$ between the SERS intensity and concentration of CAF in the Au NP: CB8 system was found (Fig. S13e, $\mathrm{ESI} \dagger)$.

\section{Multiplexed SERS sensing of drug isomers}

Furthermore, the potential multiplexed detection of structurally similar molecules using our SERS system was demonstrated with the drug isomers, TBR and TPH, at various concentrations within the $\mathrm{Au}$ NP: CB8 nanoaggregates (Fig. 5a). The main peaks of TPH and TBR, at $573 \mathrm{~cm}^{-1}$ and $1312 \mathrm{~cm}^{-1}$ respectively, can be clearly observed in all SERS spectra (Fig. 5b). Good linear correlations $\left(R^{2} \sim 0.87->0.99\right)$ 
(a)

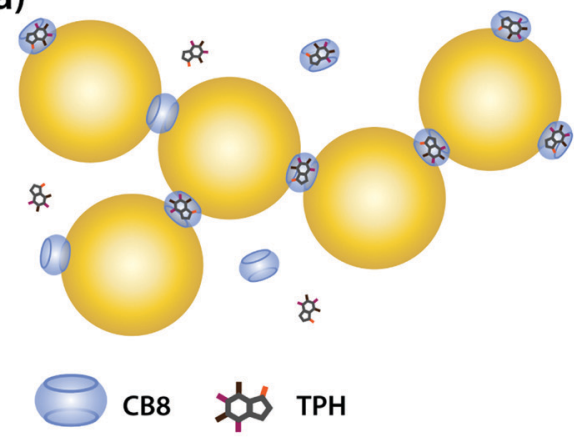

(b)

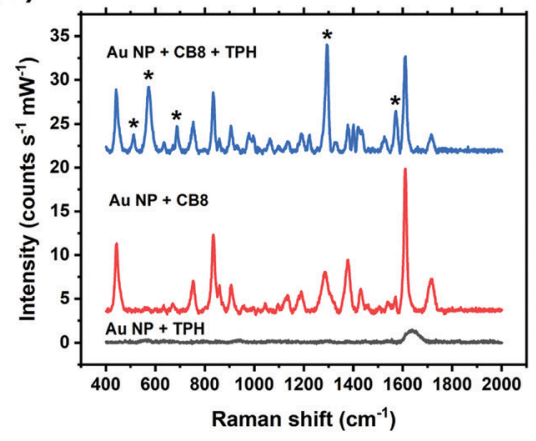

(c)

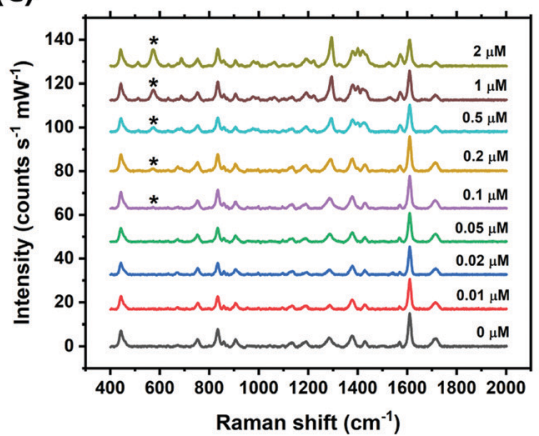

(d)

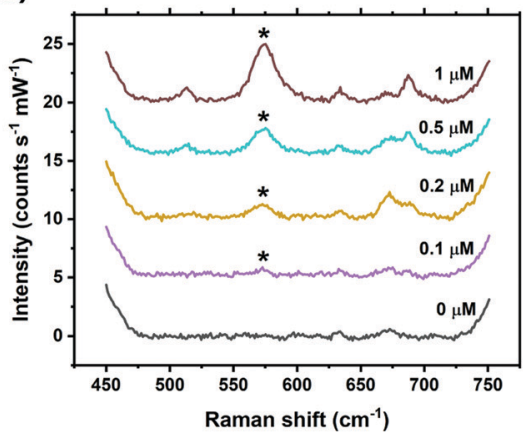

(e)

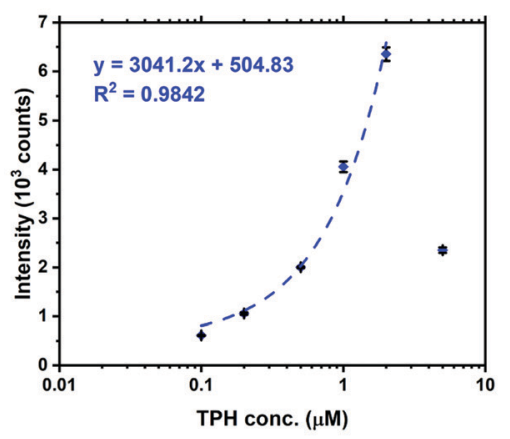

Fig. 4 (a) Schematic illustration of the precise plasmonic hotspots within Au NP: CB8 nanoaggregates for TPH detection (not to scale). (b) SERS spectra of $2 \mu \mathrm{M} \mathrm{TPH}$ in the presence or absence of CB8. (c) Full-range and (d) zoom-in SERS spectra of TPH with different concentrations from 0 to $2 \mu \mathrm{M}$. Main Raman peak of TPH at $573 \mathrm{~cm}^{-1}$ is marked by $x$. Spectra were baseline corrected and offset for clarity. (e) Corresponding plot of SERS intensity of the main TPH peak (marked by $x$ in (d)) against TPH concentration. Power-law fittings were performed to reveal correlation between SERS intensity and TPH concentration. Note: $x$-axis is plotted in log-scale to even out the spread of the data points for better illustration. The linear region at low concentration had been identified and fitted linearly.

between the SERS intensity and concentration of TBR and TPH were found for all mixtures with a combined concentration of MeX between 0.25 and $1 \mu \mathrm{M}$ (Fig. 5c). Meanwhile, the small error bars ( $\sim 1-18 \%$ error) in Fig. $3-5$ and Fig. S10, S11 (ESI $\dagger$ ) indicate the high reproducibility of the SERS signals in our sensing scheme. Notably, the multiplexed detection of MeX at sub- $\mu \mathrm{M}$ levels can be performed using Au NP-based SERS system, in contrast to precedent examples using Ag NPs, ${ }^{1,20}$ with improved detection limit. Therefore, it is possible to distinguish isomers using our SERS sensors by identifying their characteristic Raman peaks, as opposed to other techniques which do not allow molecular fingerprinting.

\section{Multiplexed quantification using machine learning techniques}

Machine learning techniques were employed in further analysing the Au NP: CB8 dataset, with an aim of tackling the nonlinear correlations at high analyte concentrations and therefore extending the detection range. It should be noted that the employed full dataset consists of all SERS spectra of TBR and TPH measured using the Au NP: CB8 system, including those of single analyte samples (TBR as shown in Fig. 3 and TPH as shown in Fig. 4) and binary mixtures (as shown in Fig. 5). Two different algorithms, partial least squared regression (PLSR) and artificial neural networks (ANNs), were used to predict the metabolite concentrations from unseen spectra
(Fig. S17A and B, ESI $\dagger$ ). The models were trained and tested via the bootstrapping random resampling procedure (Fig. S17D, ESI $\dagger$ ). A total of 1000 bootstrapping iterations were performed and the mean $R^{2}$ and root mean square error of prediction (RMSEP) values were calculated to assess the models.

At higher analyte concentrations (above $1 \mu \mathrm{M}$ ), the magnitude of the SERS peaks increases less significantly, and in some cases even decreases, due to disruption in the Au NP aggregation. To form plasmonic nanojunctions, both portals of a CB molecule need to bind to Au NPs. When host-guest complexes are formed between CB8 and TBR or TPH, one of the portals of the CB8 molecule could become partially hindered, weakening its ability to aggregate the Au NPs. At high analyte concentrations, there may not be sufficient free CB8 to mediate the fast aggregation kinetics of the Au NPs, so the SERS signals could become significantly weaker. This means that the magnitudes of the characteristic peaks are no longer linearly proportional to the analyte concentrations.

For the Au NP: CB8 dataset, the relationships between the intensity of the characteristic peaks and the analyte concentrations are linear up to $1 \mu \mathrm{M}$ and start to become nonlinear at concentrations above $1 \mu \mathrm{M}$ (Fig. S14, ESI $\dagger$ ). To assess the ability of the machine learning algorithms to model linear and nonlinear relationships, the dataset was split into two versions: one 
(a)

which contained solutions with individual analyte concentrations up to $1 \mu \mathrm{M}$ and another which included all of the solutions analysed (concentrations up to $5 \mu \mathrm{M}$ ). PLSR and ANN models were trained and tested using both versions of the dataset.

After optimisation, the number of components used in the PLSR models were 6 and 8 for the $0-1 \mu \mathrm{M}$ and $0-5 \mu \mathrm{M}$ datasets respectively and the final ANN architectures were 1167-16-128-2 and 1167-32-32-128-16-2 (Fig. S17A and B, ESI $\dagger$ ). More components/layers are required to model the nonlinear dataset as the relationship between the spectra and the analyte concentrations is more complex. The results for the different datasets and models are shown in Table 1 and Fig. S15-S18 (ESI $\dagger$ ). The partial least squares regression model performed well $\left(R^{2}=\right.$ $0.939)$ for the linear dataset $(0-1 \mu \mathrm{M})$. However, it struggled to correctly predict the analyte concentrations for the nonlinear dataset $(0-5 \mu \mathrm{M})$. This is to be expected as the PLSR algorithm is based on linear regression. In contrast, the ANNs achieved better results with $R^{2}$ values of 0.953 and 0.948 for both the $0-1 \mu \mathrm{M}$ and $0-5 \mu \mathrm{M}$ datasets respectively. The X-loadings for each component of a PLSR model built with 6 components are included in Fig. S17J (ESI $\dagger$ ). Our results are comparable to different concentrations of TBR. (b)

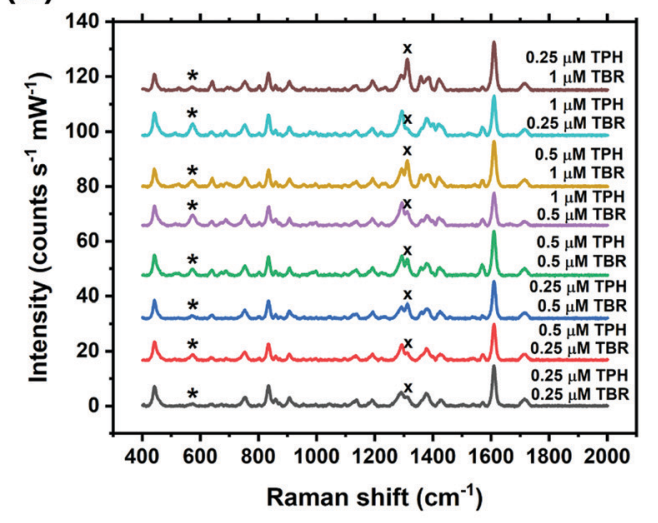

(d)

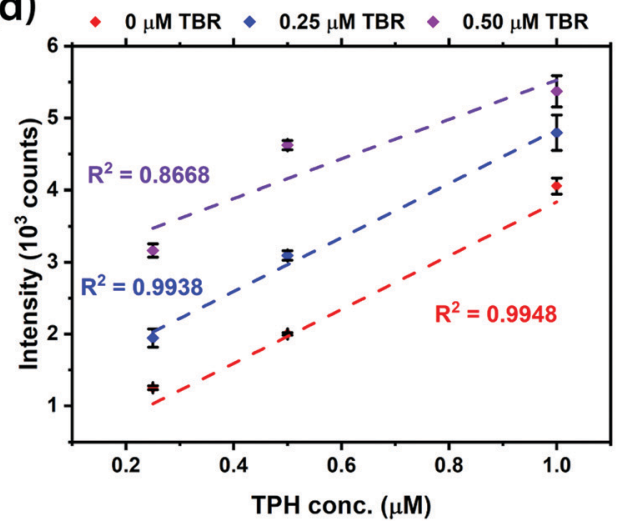

Fig. 5 (a) Schematic illustration of the multiplexed detection of TBR and TPH within Au NP: CB8 nanoaggregates (not to scale). (b) SERS spectra of corresponding plot of SERS intensity of the main TBR peak (marked by $\mathrm{x}$ in (b)) against TBR concentration in the presence of different concentrations of TPH. (d) Corresponding plot of SERS intensity of the main TPH peak (marked by * in (b)) against TPH concentration in the presence of

Table $1 R^{2}$ and root mean square error of prediction (RMSEP) values for the models. The RMSEP can also be interpreted as the detection limit

\begin{tabular}{lllllll}
\hline & & \multicolumn{2}{l}{ Theobromine } & & \multicolumn{2}{l}{ Theophylline } \\
\cline { 3 - 4 } Detection Range & & $R^{2}$ & RMSEP (M) & & $R^{2}$ & RMSEP (M) \\
\hline $0-1 \mu \mathrm{M}$ & PLSR & 0.952 & $8.22 \times 10^{-8}$ & & 0.926 & $1.02 \times 10^{-7}$ \\
& ANN & 0.968 & $6.74 \times 10^{-8}$ & & 0.939 & $9.24 \times 10^{-8}$ \\
$0-5 \mu \mathrm{M}$ & PLSR & 0.840 & $3.96 \times 10^{-7}$ & & 0.875 & $3.45 \times 10^{-7}$ \\
& ANN & 0.962 & $1.93 \times 10^{-7}$ & & 0.934 & $2.52 \times 10^{-7}$
\end{tabular}

previous work using similar techniques for SERS quantification of neurotransmitters. ${ }^{44}$

To further investigate how the algorithms interact with the spectra, PLSR and ANN models were built using spectra in which the characteristic TBR and TPH peaks had been removed (Fig. S20, ESI $\dagger$ ). The performance of these models was only slightly worse (less than a 0.035 reduction in $R^{2}$ ) than the models trained on the original spectra which contained the characteristic peaks (Fig. S21 and S22, ESI $\dagger$ ). This finding highlights the ability of the machine learning algorithms to extract subtle information from datasets and justifies using the entire spectra to make robust predictions. 
For the ANN trained on the larger dataset, the root mean square errors of prediction (RMSEPs), which can also be interpreted as the detection limits, ${ }^{1}$ were $1.93 \times 10^{-7} \mathrm{M}$ and $2.52 \times$ $10^{-7} \mathrm{M}$ for TBR and TPH, which are consistent with those determined directly by SERS titration experiments (Fig. 3d and 4d). Therefore, using the ANN model has extended the quantification range for multiplexed TBR and TPH from $1 \mu \mathrm{M}$ to $5 \mu \mathrm{M}$ without compromising the prediction accuracy. It has also enabled the simultaneous quantification of both analytes. These results are significant because they demonstrate that the Au NP: CB8 system can achieve comparable detection limits to analytical techniques such as high-performance liquid chromatography $\left(0.07 \mathrm{mg} \mathrm{L}^{-1}(0.39 \mu \mathrm{M})\right.$ for TBR and $0.08 \mathrm{mg} \mathrm{L}^{-1}$ $(0.44 \mu \mathrm{M})$ for TPH using HPLC-UV $)^{45,46}$ whilst also being portable, quicker to run and more cost-effective. ${ }^{47,48}$

\section{Conclusions}

A novel multiplexed SERS sensing platform that utilises various host-guest complexes of $\mathrm{CB} n(n=7,8)$ to detect and quantify structurally similar MeX drug molecules, TBR, TPH and CAF, has been developed. The key binding parameters of the six different 1:1 CB-MeX complexes have been quantified using NMR, UV-Vis and supported by DFT molecular models. The potential SERS detection of TBR, TPH and CAF has been demonstrated by using CB7 or CB8 to mediate the formation of precise plasmonic hotspots within the $\mathrm{Au}$ NP: CB nanoaggregates. We also showed that the binding constants can be significantly enhanced using a host-guest size matching approach. The detection limits of TBR and TPH using our system have reached $\sim 50 \mathrm{nM}$ with highly reproducible SERS signals, as opposed to the relatively weaker signals of CAF due to its larger size. The capability of our SERS system to simultaneously quantify multiple structurally similar molecules has also been successfully demonstrated with the drug isomers, TBR and TPH, in proof-of-concept experiments, where the dynamic range of the sensor can be extended using machine learning algorithms. Hence, our SERS sensor holds great potential for a wide range of applications including therapeutic drug monitoring, food processing, forensics and veterinary science.

\section{Conflicts of interest}

There are no conflicts to declare.

\section{Acknowledgements}

TCL is grateful to the support from the Research Project Grant (RPG-2016-393) funded by the Leverhulme Trust, the Royal Society Research Grant 2016 R1 (RG150551) and the UCL BEAMS Future Leader Award funded through the Institutional Sponsorship award by the EPSRC (EP/P511262/1). WIKC, IPP, MO and TCL are grateful to the Studentship funded by the A*STAR-UCL Research Attachment Programme through the EPSRC M3S CDT (EP/L015862/1). TJ and TCL acknowledge Camtech Innovations for contributing to TJ's studentship.
WIKC, JK, DUS and MO thank IBB TBL for use of facilities and resources. JP, DUS and MO would like to acknowledge the support from A*STAR BMRC Central Research Fund (UIBR). We acknowledge the use of the UCL Thomas high performance computing facility (Thomas@UCL), and the associated support services, for the completion of this work.

\section{References}

1 O. Alharbi, Y. Xu and R. Goodacre, Anal. Bioanal. Chem., 2015, 407, 8253.

2 M. E. Rybak, M. R. Sternberg, C.-I. Pao, N. Ahluwalia and C. M. Pfeiffer, J. Nutr., 2015, 145, 766.

3 E. Martinez-Pinilla, A. Onatibia-Astibia and R. Franco, Front. Pharmacol., 2015, 6, 30.

4 P. Sanphui and A. Nangia, J. Chem. Sci., 2014, 126, 1249.

5 N. Sugimoto, S. Miwa, Y. Hitomi, H. Nakamura, H. Tsuchiya and A. Yachie, Nutr. Cancer, 2014, 66, 419.

6 M. S. Bispo, M. C. C. Veloso, H. L. C. Pinheiro, R. F. S. De Oliveira, J. O. N. Reis and J. B. De Andrade, J. Chromatogr. Sci., 2002, 40, 45.

7 P. Holmgren, L. Norden-Pettersson and J. Ahlner, Forensic Sci. Int., 2004, 139, 71.

8 C. Cortinovis and F. Caloni, Front. Vet. Sci., 2016, 3, 26.

9 C. W. Huck, W. Guggenbichler and G. K. Bonn, Anal. Chim. Acta, 2005, 538, 195.

10 M. R. Brunetto, L. Gutierrez, Y. Delgado, M. Gallignani, A. Zambrano, A. Gomez, G. Ramos and C. Romero, Food Chem., 2007, 100, 459.

11 J. J. Carvalho, M. G. Weller, U. Panne and R. J. Schneider, Anal. Bioanal. Chem., 2010, 396, 2617.

12 N. Chiem and D. J. Harrison, Anal. Chem., 1997, 69, 373.

13 V. K. Gupta, A. K. Jain and S. K. Shoora, Electrochim. Acta, 2013, 93, 248.

14 Y. Peng, W. Zhang, J. Chang, Y. Huang, L. Chen, H. Deng, Z. Huang and Y. Wen, Food Anal. Methods, 2017, 10, 3375.

15 F. Bates and M. del Valle, Microchim. Acta, 2015, 182, 933.

16 W. Xu, T.-H. Kim, D. Zhai, J. C. Er, L. Zhang, A. A. Kale, B. K. Agrawalla, Y.-K. Cho and Y.-T. Chang, Sci. Rep., 2013, 3, 2255.

17 A. K. Mahapatra, P. Sahoo, S. Goswami, S. Chantrapromma and H.-K. Fun, Tetrahedron Lett., 2009, 50, 89.

18 P. Liu, R. Liu, G. Guan, C. Jiang, S. Wang and Z. Zhang, Analyst, 2011, 136, 4152.

19 H. Zheng, D. Ni, Z. Yu, P. Liang and H. Chen, Sens. Actuators, B, 2016, 231, 423.

20 M. Wu, H. Li, D. Lv and F. Lu, Anal. Bioanal. Chem., 2017, 409, 5569.

21 J. Kang, H. Gu, L. Zhong, Y. Hu and F. Liu, Spectrochim. Acta, Part A, 2011, 78, 757.

22 I. Pavel, A. Szeghalm, D. Moigno, S. Cinta and W. Kiefer, Biopolymers, 2003, 72, 25.

23 R. Hu, R. Tang, J. Xu and F. Lu, Anal. Chim. Acta, 2018, 1034, 176.

24 Y. Kurokawa, Y. Imai and Y. Tamai, Analyst, 1997, 122, 941. 
25 W. W. Y. Lee, V. A. D. Silverson, C. P. McCoy, R. F. Donnelly and S. E. J. Bell, Anal. Chem., 2014, 86, 8106.

26 X. Chen, H. Gu, G. Shen, X. Dong and J. Kang, J. Mol. Struct., 2010, 975, 63.

27 R. Sato-Berru, R. Redon, A. Vazquez-Olmos and J. M. Saniger, J. Raman Spectrosc., 2009, 40, 376.

28 H.-H. Jeong, E. Choi, E. Ellis and T.-C. Lee, J. Mater. Chem. B, 2019, 7, 3480.

29 M. Velicka, S. Adomaviciute, E. Zacharovas and V. Sablinskas, Proc. SPIE, 2020, 11257, 112570S.

30 T.-C. Lee and O. A. Scherman, Chem. Commun., 2010, 46, 2438.

31 T.-C. Lee and O. A. Scherman, Chem. - Eur. J., 2012, 18, 1628.

32 R. W. Taylor, T.-C. Lee, O. A. Scherman, R. Esteban, J. Aizpurua, F. M. Huang, J. J. Baumberg and S. Mahajan, ACS Nano, 2011, 5, 3878.

33 W.-I. K. Chio, W. J. Peveler, K. I. Assaf, S. Moorthy, W. M. Nau, I. P. Parkin, M. Olivo and T.-C. Lee, J. Phys. Chem. C, 2019, 123, 15769.

34 W.-I. K. Chio, S. Moorthy, J. Perumal, U. S. Dinish, I. P. Parkin, M. Olivo and T.-C. Lee, J. Mater. Chem. C, 2020, 8, 7051.

35 W.-I. K. Chio, G. Davison, T. Jones, J. Liu, I. P. Parkin and T.-C. Lee, J. Visualized Exp., 2020, 164, e61682.

36 J. Wu and L. Issacs, Chem. - Eur. J., 2009, 15, 11675.
37 J. Lagona, P. Mukhopadhyay, S. Chakrabarti and L. Issacs, Angew. Chem., Int. Ed., 2005, 44, 4844.

38 W. A. Ameku, W. R. de Araujo, C. J. Rangel, R. A. Ando and T. R. L. C. Paixao, ACS Appl. Nano Mater., 2019, 2, 5460.

39 S. Mecozzi and J. Rebek, Chem. - Eur. J., 1998, 4, 1016.

40 T.-C. Lee, E. Kalenius, A. I. Lazar, K. I. Assaf, N. Kuhnert, C. H. Grun, J. Janis, O. A. Scherman and W. M. Nau, Nat. Chem., 2013, 5, 376.

41 H. G. M. Edwards, T. Munshi and M. Anstis, Spectrochim. Acta, Part A, 2005, 61, 1453.

42 S. Gunasekaran, G. Sankari and S. Ponnusamy, Spectrochim. Acta, Part A, 2005, 61, 117.

43 S. Mahajan, T.-C. Lee, F. Biedermann, J. T. Hugall, J. J. Baumberg and O. A. Scherman, Phys. Chem. Chem. Phys., 2010, 12, 10429.

44 S. Kasera, L. O. Herrmann, J. D. Barrio, J. J. Baumberg and O. A. Scherman, Sci. Rep., 2014, 4, 6785.

45 B. Srdjenovic, V. Djordjevic-Milic, N. Grujic, R. Injac and Z. Lepojevic, J. Chromatogr. Sci., 2008, 46, 144.

46 F. Buiarelli, F. Bernardini, G. Simonetti, P. Di Filippo, D. Pomata, C. Riccardi and R. Risoluti, J. AOAC Int., 2019, 102, 865.

47 M. W. Dong, Modern HPLC for Practicing Scientists, John Wiley \& Sons, 2006.

48 J. A. Dietrich, A. E. McKee and J. D. Keasling, Annu. Rev. Biochem., 2010, 79, 563. 